\title{
Tracking the emergence of new SARS-CoV-2 variants in South Africa
}

\author{
A large genomic surveillance effort in South Africa produced 1,365 high-quality whole genomes of SARS-CoV-2; \\ this has identified 16 new lineages of the virus that had not been identified in other countries.
}

\author{
Anise N. Happi, Chinedu A. Ugwu and Christian T. Happi
}

A s of 19 January 2021, there had been over 96 million reported cases of COVID-19 and 2 million reported deaths ${ }^{1}$. Since the beginning of the COVID-19 pandemic, genomic surveillance has been playing a pivotal role in the public-health interventions of many countries across the globe. Sequences of the coronavirus SARS-CoV-2 from various parts of the world have been continuously deposited in real time on the GISAID database $\mathrm{e}^{2}-$ a global science initiative and primary source established in 2008 that provides open access to viral genomic data and is freely and easily accessible. At the time of this writing, about 387,000 high-quality sequences of SARS-CoV-2 have been generated from the current COVID-19 pandemic across the globe ${ }^{2}$, and over 320 variants and/or lineages of SARS-CoV-2 have been identified worldwide ${ }^{3}$. In this issue of Nature Medicine, Tegally et al. elegantly demonstrate how African scientists are leveraging the power of genomics in near real time to monitor the COVID-19 outbreak in South Africa ${ }^{4}$.

In response to the spread of SARS-CoV-2, genomics experts in South Africa have come together to form the Network for Genomics Surveillance in South Africa. In the new study ${ }^{4}$, they utilize genomic tools to monitor the evolution and dynamics of the SARS-CoV-2 in South Africa from the first introduction of the virus in March 2020 to September 2020. The researchers have been able to generate 1,365 high-quality SARS-CoV-2 genomes. 42 lineages or variants of SARS-CoV-2 have been identified across eight provinces in South Africa, of which 16 variants seem to be specific to that country. 3 of the 16 variants (variants C. 1 , B.1.1.54 and B.1.1.56) became dominant, accounting for about $42 \%$ of the total generated sequences.

A variant or lineage is a virus that has acquired some genetic changes (mutation) relative to the parental virus and shows

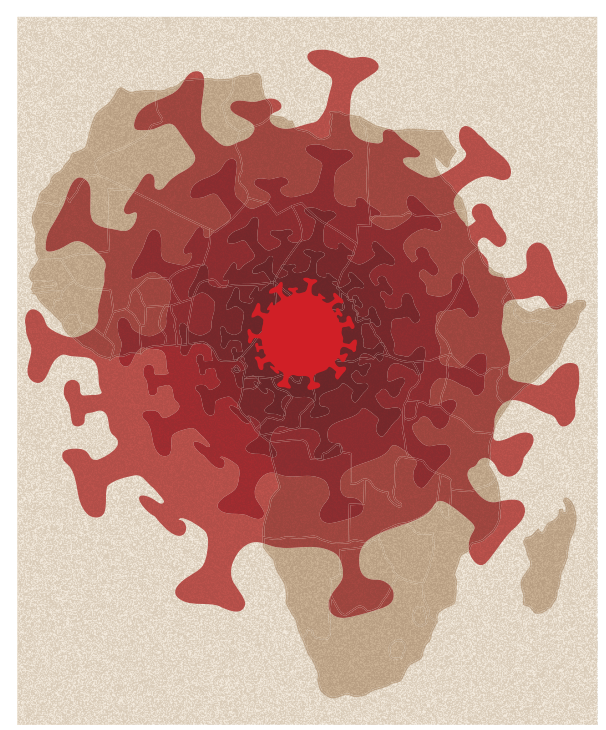

Credit: Andrei Krauchuk / Alamy Stock Vector

evidence of ongoing transmission in a geographically distinct region. These genetic changes can make the new variants behave differently from the parental virus in terms of spread and hospitalization and may affect the efficacy of vaccines and diagnoses. For example, the B1.1.7 variant that was first identified in the UK has been associated with increased viral spread and hospitalization ${ }^{5}$. Genetic changes can also be silent and have no effect on the behavior of the virus. The implication of the emergence and dominance of some of these variants in terms of viral transmission, hospitalization, and diagnostic and vaccine efficacy is only beginning to be ascertained.

In the new study, Tegally et al. use a phylogeographic model to understand the location, time and spread of these three major lineages, C.1, B.1.1.54 and B.1.1.56, across different provinces in South Africa ${ }^{4}$. Data from the study also reveal that the closure of international borders resulted in a reduction in the influx of viral lineages from outside the country. Locally, specific lineages emerged, with three lineages dominating the viral population, during the period when the national lockdown was eased from level 4 to level 3. Thus, a ban on international travel is likely to have fueled the emergence of the South Africa-specific variants of SARS-CoV-2, with the increased internal movement of people and the easing of lockdown allowing the dominance of these local variants. Another driver of the emergence of the new lineages appears to be nosocomial infections. Lineage B.1.106 emerged in April 2020 after a hospital outbreak and was responsible for $45 \%$ of the deaths due to COVID-19 at that time. Finally, using a genomic tool combined with active epidemiology data, the authors find that the hospital outbreak was controlled and the SARS-CoV-2 variants in the hospital population were reduced within 2 months.

The emergence of different variants of SARS-CoV-2 across the globe is worrisome, especially as the mutations acquired by these variants have the potential to increase viral transmission and to affect clinical outcomes and diagnostic and vaccine efficacy $^{6}$. SARS-CoV-2 variants have also been reported in other African countries, including Nigeria ${ }^{7}$. Although Tegally et al. find that the genetic changes acquired by the three South Africa-dominant variants have not conferred a fitness advantage to them ${ }^{4}$, more data combining clinical, pathological and epidemiological information are needed for better understanding of the impact of these new variants on disease dynamics. There is now emerging evidence that some of the new SARS-CoV-2 variants could potentially escape the immune system, with downstream effects on the efficacy of the few available vaccines against COVID-198. Despite these challenges, near-real-time genomics epidemiology provides a unique opportunity to gather information for the quick development of alternative or modified public-health countermeasures. 
Genomic surveillance is becoming a core part of public-health intervention. It is providing real-time data on viral evolution and transmission dynamics that will aid public-health policy formulation, diagnostics and vaccine design. The Africa Centre of Excellence for Genomics of Infectious Disease at Redeemer's University in Ede, Nigeria, generated the first SARS-CoV-2 sequence from Africa within 72 hours of receipt of the sample ${ }^{9}$ and thus helped Nigerian health authorities to understand the imported origin of the virus. The Africa Centre of Excellence for Genomics of Infectious Disease, in partnership with the Broad Institute, is leading a new and unique public-health surveillance system called 'Sentinel'. This new initiative utilizes ultra-sensitive genomic technologies to develop an early-warning system for preempting pandemics and to detect viral threats in real time (https://audaciousproject. org). The Sentinel system is being piloted in West and Central Africa. Other initiatives, such as the Network for Genomics Surveillance in South Africa, the Public Health Alliance for Genomic Epidemiology in South Africa, and the Pathogen Genomic Initiative by the Africa Centre for Disease Control and Prevention, are working to integrate genomics into public-health surveillance on the continent ${ }^{10}$. Despite the limited resources of the continent, African scientists are rising to the challenge, showing how genomic sequencing data can be used in settings with meager resources to enable targeted public-health interventions during an outbreak.

\section{Anise N. Happi (D)1, Chinedu A. Ugwu(D)1,2 and Christian T. Happi (D) $1,2 \otimes$ \\ ${ }^{I}$ African Center of Excellence for Genomics of Infectious Disease, Redeemer's University, Ede, Nigeria. ${ }^{2}$ Department of Biological Sciences, Faculty of Natural Sciences, Redeemer's University, Ede, Nigeria.

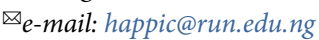

Published online: 15 March 2021

https://doi.org/10.1038/s41591-021-01265-1

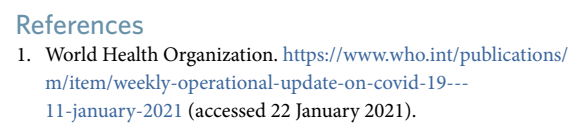

2. Shu, Y. \& McCauley, J. Euro. Surveill. 22, 2-4 (2017).

3. PANGO lineages. https://cov-lineages.org/descriptions.html (2021).

4. Tegally, H. et al. Nat. Med. https://doi.org/10.1038/s41591-02101255-3 (2021).

5. Rambaut, A. et al. Virological https://virological.org/t/ preliminary-genomic-characterisation-of-an-emergent-sars-cov2-lineage-in-the-uk-defined-by-a-novel-set-of-spikemutations/563 (2020).

6. Centers for Disease Control and Prevention. https://www.cdc. gov/coronavirus/2019-ncov/more/scientific-brief-emergingvariant.html (accessed 22 January 2021).

7. Happi, C., Oluniyi, P., Olawoye, I., Ihekweazu, C. \& Nkengasong, J. Virological https://virological.org/t/detection-ofsars-cov-2-p681h-spike-protein-variant-in-nigeria/567 (2020).

8. Constantinos, K.W. et al. Preprint at bioRxiv https://doi. org/10.1101/2021.01.18.427166 (2021).

9. Happi, C. et al.. Virological https://virological.org/t/first-africansars-cov-2-genome-sequence-from-nigerian-covid-19-case/421 (2020).

10. Tessema, K. S. et al. Lancet Microbe 1, e227-e228 (2020).

\section{Acknowledgements}

This work is supported by the BBSRC-OVEL project BB/R020116/1 and Wellcome Trust project 216619/Z/19/Z; The World Bank (ACE019 and

ACE-IMPACT); and the US National Institutes of Health (grant U54HG007480). C.T.H. is also supported by the ELMA, Flu Lab and the Skoll Foundation through the TED Audacious Project.

\title{
Understanding life and death in Latin America
}

\author{
An analysis of life expectancy and cause of death in 363 Latin American cities unveils wide differences between \\ cities in the same country, and sheds light on the socioeconomic factors that determine how long people live and \\ how they die.
}

\section{Fernando Lanas and Pamela Seron}

O ver the past several decades, life expectancy has increased more than during any other period of human existence, and global inequality in life expectancy was reduced between 1970 and 2000 (ref. ${ }^{1}$ ). Unfortunately, life expectancy still differs widely between countries and between socioeconomic group ${ }^{2,3}$. Now, in Nature Medicine, Bilal et al. present the initial results of SALURBAL (Salud Urbana en América Latina), an international project aimed at studying how urban environments and urban policies affect the health of city residents throughout Latin America ${ }^{4}$. They find that important differences exist between larger cities in Latin America, not only in life expectancy but also in causes of death (Fig. 1).

The SALURBAL project used a harmonized dataset to assess variability and predictors of life expectancy and proportionate mortality in 363 cities with a population above 100,000 people in 2010, across nine Latin American countries: Argentina, Brazil, Chile, Colombia, Costa Rica, El Salvador, Mexico, Panama, and Peru. Life expectancy varied between countries, with cities in Panama, Chile, and Costa Rica reporting the longest life expectancy, and cities in Mexico, Brazil, Peru, and El Salvador at the opposite extreme, with the shortest life expectancy. Notably, life expectancy differed substantially across cities within the same country-up to 8 years for women, and up to 14 years difference for men. In comparison, disparities in the average life span reach 30 years of difference between the world's longest-lived countries, with life expectancy at birth above 84 years in 2019
(Japan, Hong Kong, and Macao), and the shortest-lived countries, with life expectancy at birth below 55 years (Central African Republic, Chad, Lesotho, Nigeria, and Sierra Leone $)^{2}$.

Many factors have been described as being determinants of life expectancy, including lifestyle, social and economic status, education, employment, housing, physical and environmental exposures ${ }^{5}$, risk-factor prevalence, accessibility of health facilities, and quality of health services ${ }^{6}$. The impact of these factors varies according to the economic-development status of the country. Investigators for the PURE (Prospective Urban Rural Epidemiology) study have reported marked differences between countries of different income in the determinants of mortality, in a cohort of 182,375 people. Household air pollution, 